\title{
Supporting Scenario Building in Product Design
}

\author{
Irene Anggreeni \\ Laboratory of Design, Production \& Management, \\ University of Twente \\ PO Box 217, 7500 AE Enschede, \\ The Netherlands \\ +31 (0)534893192 \\ i.anggreeni@utwente.nl
}

\author{
Mascha van der Voort \\ Laboratory of Design, Production \& Management, \\ University of Twente \\ PO Box 217, 7500 AE Enschede \\ The Netherlands \\ +31 (0)53 4892520 \\ m.c.vandervoort@utwente.nl
}

\begin{abstract}
Designing consumer products has grown more challenging due to the increasingly complex characteristics of these products. Design research has promoted the use of scenarios -concrete stories about product use- to support the design process. However, design practice still lacks a thorough guidance that integrates various scenario uses effectively. In this paper, we describe a software concept that guides designers in generating meaningful scenarios. Inspired by design practice, we expect the support tool to serve as a framework for documenting design knowledge, enabling designers to make well-informed decisions.
\end{abstract}

\section{Categories and Subject Descriptors}

H.4.2 [Information Systems Application]: Types of System decision support.

\section{General Terms}

Design, Documentation, Human Factors, Management.

\section{Keywords}

Scenario based product design, user centered design, consumer product, scenario building, support tool, software.

\section{INTRODUCTION}

Designing consumer products has been a growing challenge for design companies. Due to the competitive market, designers need to convince the potential end-users that their product is the best value for money. Often, this is achieved by (over)loading the product with many functions which aim to accommodate various users in different settings. Therefore, we can observe a trend in design for usability of consumer products by taking into account their dynamic use situations [3]. These characteristics demand designers to be aware of all the necessary and relevant information to make sound design decisions. Concrete stories could help glue together the information pieces and make them more comprehensible. These stories are referred to as 'scenarios' that the approach of applying scenarios in a design process is known as 'scenario based design' (SBD).

\footnotetext{
(c) The Author 2008.
}

Published by the British Computer Society
Originating from the software engineering discipline, SBD helps to tackle technical problems especially in the activities of balancing reflections and actions, supporting participation of stakeholders, and building repository of design knowledge [4]. With more software applications embedded in every day products, the same benefits of using scenarios could as well be applicable in the design of consumer products. There have been initiatives to study the application of scenarios for designing user-friendly consumer products, creating a new movement of 'scenario based product design' (SBPD). There exist scenario based approaches to conduct activities common in product design (e.g. [6, 8]). However, design practices still lack a thorough guidance that integrates different scenario uses over a design process. Additionally, a concrete framework on the scenario building activities is necessary to make the approaches more applicable in practice. Without such guidance, designers would face doubts whether they have identified, created and communicated scenarios in an optimal way.

Design companies are often discouraged by the uncertainties involved in applying scenario based approaches despite the promising benefits. Scenarios are currently being used for different purposes which are often applicable only to specific design activities. To get an overview of the many ways scenarios can support a design process, a classification of scenarios based on their usages was pursued [1]. Overall, scenarios can be applied in a design process in six categories of use, where each use involves a specific type of scenario. We have identified that the six scenario categories provides a good coverage on the design phases in which designers explore the design domain, (re)define focused problems and solutions, and validate their solutions. By relating scenarios to their proper positions in user centered design, we hope to clarify that SBPD should not be a total reconstruction of a design practice. Rather, scenarios are able to offer support in the loose ends of design activities.

The main activity in SBPD, the scenario building, extends normal design inquiries. Scenario building is a productive synthesis that gathers information pieces acquired during analysis into informal design knowledge. This knowledge in turn, provides designers clear references to reason about their design. To guide the process of scenario building, we propose a tool that aids designers in documenting information pieces, integrating them into scenarios, and representing the scenarios to the stakeholders.

This paper presents the preliminary requirements for the scenario support tool which are synthesized from two workshops with designers. A concept for the tool is formulated and will be described as a result of this research. 


\section{APPROACH}

This research aims to support design practice by means of a scenario building support tool. While frameworks to develop computer support for scenario based design already exist, they often focus on specific areas such as the modeling of design requirements (e.g. [5, 7]). To make sure that this research contributes to design practice, the activity of designing with scenarios was studied both from theoretical as well as practical point of view. As described, an overview of scenario uses in design-related domains has been formulated in a scenario use classification [1]. This scenario use classification functioned as a common reference for discussions with designers about their scenario practice and the challenges related to it. A workshop series was conducted to learn and understand how designers do or would use scenarios in ways that fit their practice. The workshops result in an overview of some practical design challenges in which scenarios could be useful. Consequently, the workshop experience also contributes to the development of preliminary requirements for the scenario support tool. More workshops are currently planned to examine design practice in other companies as well as to verify the results from the previously performed workshops.

The next section will elaborate our workshop findings so far. Based on these findings, a set of preliminary requirements is formulated and used to sketch out the concept of the support tool. This concept envisions the improvements in design activities that can be achieved by the support tool.

Our approach involves an active role of designers in the concept development of the support tool. Successive workshop series will be conducted to achieve this. Eventually, a practical evaluation of this research will be based on how useful designers find the support tool in their practice and how helpful it is in aiding better design thinking.

\section{RESULTS}

At the time of writing, two workshops have been performed with designers at a Dutch medium-sized design company. The participating designers all had some experience in using scenarios. In each workshop, two designers were presented with the classification of scenario uses. Our workshop objectives then were threefold: (1) to evaluate the scenario use classification from a practical point of view, (2) to get insight in designers' practice and the potentials of using scenarios in their work, and (3) to discuss forms of support applicable in their design practice. A fictional case study of designing a bicycle baggage carrier was used to simulate a design project, giving concrete points for discussion with the participants.

\subsection{Workshop Findings}

The performed workshops gave an overall idea of design practice at the company (henceforth, company X). Our scenario use classification was used as a common reference to bridge the theoretical SBPD and the X-designers' scenario practice. The classification was confirmed for its coverage within X's specific design process. All current and envisioned scenario uses mentioned by the participating designers could relate to our classified scenario types. The next paragraph summarizes the design practice as discovered during the workshops at $\mathrm{X}$.

Designers at $\mathrm{X}$ build their initial design knowledge based on contact with end-users, observations of current use of competitive products and applicable established standards (e.g. ISO). Short scenarios are used to register the design knowledge and later to validate the designs in quick iterations (i.e. without inviting users in formal testing sessions). These critical use scenarios -as the designers call them- focus on all possible situations in which their product can fail in its full life cycle, starting from production to disposal or recycle. The designers run through all or parts of the critical use scenarios during certain stages of their design progress. Often, this is done with fellow designers to ensure more sound judgment. On the other hand, the explicit use of scenarios to highlight design problems and generate design ideas is not yet practiced. Nevertheless, the participants expressed that they actually already 'think in scenarios' while designing and self-reviewing their designs. Due to time pressure, these 'scenarios' are used in an ad-hoc way or sometimes not expressed at all. Furthermore, the critical use scenarios, though they are made explicit in documents, are often not complete. This leaves some parts of the scenarios open to assumptions, and could risk in a misunderstanding on how the users would actually perceive, react and feel in some critical situations.

An ideal practice would be to put forward all critical use scenarios as early as possible with the involvement of all stakeholders. The scenario list should then be maintained as the design project progresses. However in practice, designers often document sporadically whenever they have spare time apart from designing. Documenting is seen as obstructions to their creativity. On the other hand, designers also realize the need of adapting a more structured approach, especially concerning the scenario usage.

From these workshop findings, we could withdraw some requirements for the support tool as the followings. Designers need support in gathering, registering and organizing relevant design information for building scenarios. Since their main challenge is getting the essence of abundant information, designers would also value a way to efficiently documenting scenarios. An overview of scenarios will become important for the designers along with the growing number of scenarios being covered. Additionally, a means to communicate scenarios quickly to the audience (e.g. users, clients, managers, etc) is necessary to make them practically useful. Finally, even with all the requirements fulfilled, the functionality of the support tool needs to be an integrated part of design activities to make it acceptable to designers.

\subsection{Scenario Building Support Tool}

Scenario building is a synthesis activity that wraps up design inquiries, a necessary part of user centered design. To support scenario building, a sensible target is its first-base activity: the gathering of necessary information as elements of the scenarios. Based on the needs that were expressed by the participating designers at $\mathrm{X}$, the support tool should cover the activities of documenting information pieces -elements of scenarios, guiding the creation of scenarios and eventually communicating them. The following subsections propose the functionality of the support tool. Some illustrations are presented to show the hypothetical use of the tool, following the fictional design case of (re)designing a bicycle baggage transporter.

\subsubsection{Inquiry into current use situations}

A dialogue model is proposed to involve all kinds of stakeholders and capture their thoughts and opinions. This dialogue can either be acted collaboratively with users or internal among designers to analyze observation results, e.g. video recordings from observations. We imply by using a dialogue model that at least another person -either user or fellow designer- is present to ensure that assumptions can be 
challenged and clarified immediately. During an inquiry process, it can also happen that the participants or the (inexperienced) inquirer or both become distracted and drifted away from the main topics. As a result, some important situations could be overlooked. The tool could help by prompting the inquirer proper questions one at a time (Figure 1 ), and by doing so, guiding the collection of complete information pieces for the scenarios.

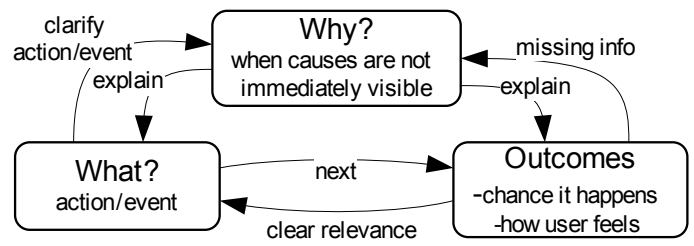

Figure 1. A guideline for questioning during an inquiry.

Thus, a hypothetical dialogue between designer(s) and a user guided by the support tool could happen as follows, taken that a common use of a bicycle baggage transporter is to "transport groceries". The interviewed user is John, an 18 year old university student.

Table 1. A dialogue example between a designer and a user.

\begin{tabular}{|l|l|}
\hline Dialogue & Goal \\
\hline $\begin{array}{l}\text { Designer: How do you transport } \\
\text { groceries on your bike? }\end{array}$ & clarifying context \\
$\begin{array}{l}\text { John: Well, when I have 2 plastic } \\
\text { shopping bags, my (elastic) straps } \\
\text { become useless. So I use my handlebar } \\
\text { to hang the bags. }\end{array}$ & \\
\hline $\begin{array}{l}\text { D: What can happen then? } \\
\text { J: Often the bags make me unbalanced. }\end{array}$ & $\begin{array}{l}\text { probing for potential } \\
\text { failures }\end{array}$ \\
\hline $\begin{array}{l}\text { D: Why does that happen? } \\
\text { J: Well, the bags would swing around } \\
\text { when I turn or run through road bumps. }\end{array}$ & investigating \\
\hline $\begin{array}{l}\text { D: What could happen then? } \\
\text { J: Nothing, I'm pretty good at biking... } \\
\text { Or I might as well fall and cause a little } \\
\text { accident. }\end{array}$ & $\begin{array}{l}\text { knowing the possible } \\
\text { they happen }\end{array}$ \\
\hline $\begin{array}{l}\text { D: What could be the worst possible } \\
\text { outcomes? }\end{array}$ & $\begin{array}{l}\text { probing the worst-case } \\
\text { effects from the user's } \\
\text { perspective }\end{array}$ \\
$\begin{array}{l}\text { J: I fall, maybe get hurt and need to be } \\
\text { hospitalized. And my groceries } \\
\text { damaged. }\end{array}$ & \\
\hline $\begin{array}{l}\text { D: How bad are those in score 1 to 10? } \\
\text { J: Me being hospitalized, that's quite } \\
\text { bad, I'd score it 6. While my damaged } \\
\text { groceries, just a 3. }\end{array}$ & $\begin{array}{l}\text { an indication needed, } 1 \text { is just a slight } \\
\text { annoyance, 4 is 'I am } \\
\text { never going to use it } \\
\text { again', etc }\end{array}$ \\
\hline
\end{tabular}

The example shows how a dialogue could reveal personal uses of products, what matters to users and in which ways the current products fail to satisfy them.

\subsubsection{Structuring information from inquiries}

Automatic building of cause-and-effect networks is proposed to structure the information pieces, making them more accessible later on. The proposed functionality helps structure information into a knowledge representation that comprises scenario storylines. Figure 2 shows a network of information that represents this knowledge.

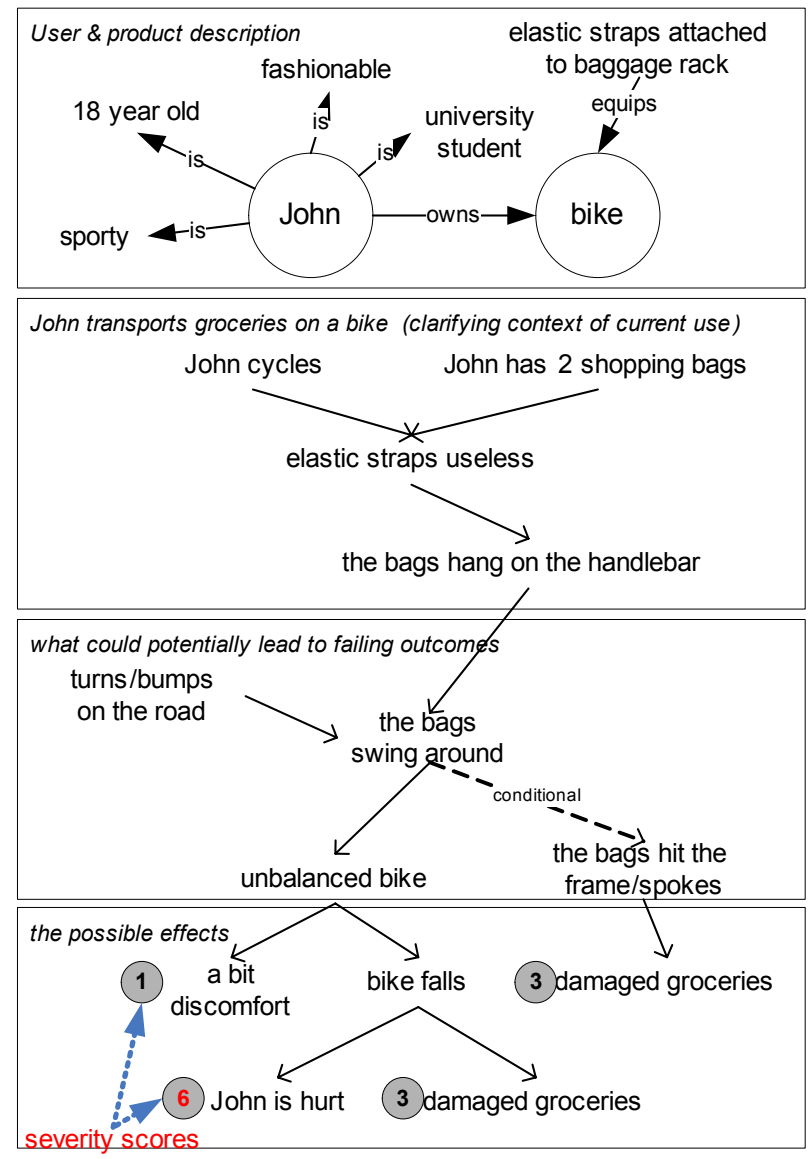

Figure 2. A construction of cause-and-effect networks using information gathered during inquiries.

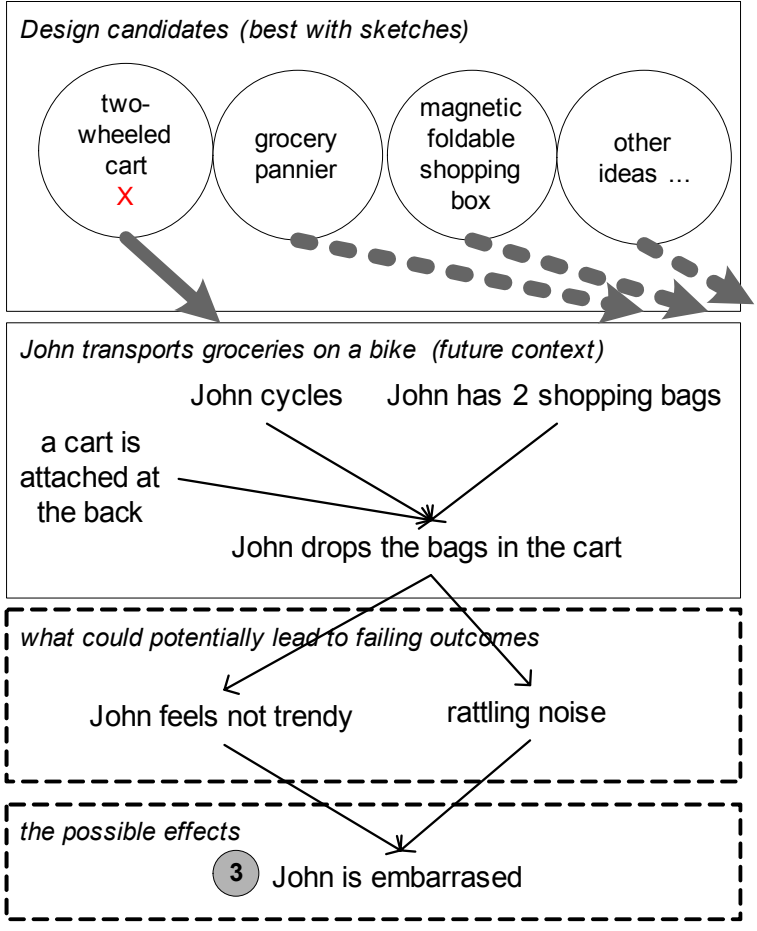

Figure 3. A quick evaluation of design candidates with the user, using similar cause-and-effect networks. 
Furthermore, the design team could also put forward other technologies or design ideas and immediately get feedback why they would or would not fit in the users' world. Figure 3 shows an evaluation of preliminary design ideas with the user. It is not present in the dialogue example (Table 1), but included here to give an idea about the evaluation of several design candidates based on users' rationales.

\subsubsection{Guiding the creation of scenarios}

By creating coherent scenarios, the designers can focus on the most essential information to guide their decisions. Scenarios can be easily constructed once the storylines are available within the cause-and-effect networks. With various storylines being registered, designers can make well-informed selection of scenarios that highlight key issues by looking up how the users feel about it (from the given severity scores). The existing storylines give flexibility to represent scenarios in different media (e.g. text, storyboard, cartoon and role play). The following example shows a possible scenario constructed from the storyline in Figure 2.

John is an 18 year old university student. He represents the young people of his age: fashionable, spontaneous and sporty. He owns a bike that takes him anywhere in the town. His bike is equipped with elastic straps at the baggage rack.

After college, John goes to a supermarket on his bike. He ends up with 2 plastic shopping bags to carry. The elastic straps are useless in this situation, so John hangs the 2 bags on the bike handlebar. The bags swing around uncontrollably on the bumpy road. His bike becomes unbalanced and then he falls. John is hurt and his groceries are damaged.

Scenarios make explicit the design team's understanding about the users and their context, making it possible to share and verify this understanding with other stakeholders. Later on, walking through product ideas in scenarios with the users could reveal potential problems around the design ideas early.

\section{DISCUSSION AND FUTURE WORK}

Company $\mathrm{X}$ currently employs a formal risk analysis framework to support their practice of rigorous testing. We could relate this practice with extreme programming $(X P)$ [2] which emerges from the software development discipline. The idea of XP is to first and foremost build test cases as complete as possible, and later on write software codes that should pass all these test cases. The XP test cases spring from requirements, which are derived from user stories. By giving the developers all test cases up front shorter iterations can be achieved; thus $\mathrm{XP}$ is often addressed as agile software development. Relating $\mathrm{XP}$ to product design, many similarities between XP and the practice at company $\mathrm{X}$ are found, specifically in the use of scenarios for rapid iterative testing.

To adapt this approach to the product design domain, we need to understand designers' thinking process concerning their design and evaluation activities. The formal framework being used at company $\mathrm{X}$ does offer benefits, yet can be quite exhausting due to the large amount of data. Moreover, a formal framework could be a hindrance to creativity. On the other hand, a support tool that guides designers' thinking process could help release some burdens off their mind. We expect this will result in their creativity becoming more streamlined since the tool could 'watch over' the designers from derailing to a wrong path. The scenarios then become the forces that drive a design process towards solutions, as shown in Figure 4.

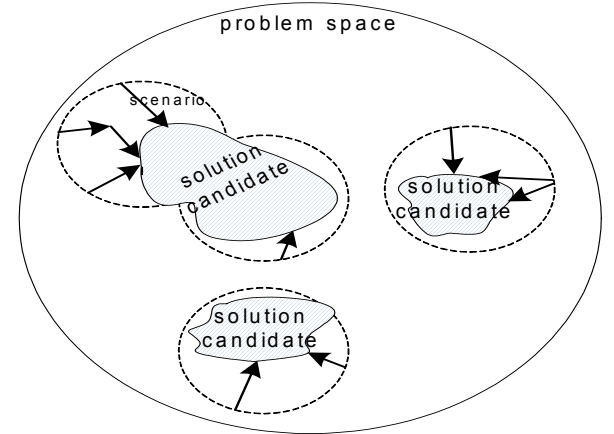

Figure 4. Scenarios drive the shaping of potential solutions systematically.

The concept of scenario building support tool presented here is still a preliminary idea. Therefore, our future work includes exploration and maturation of the scenario building support concept. Although we encountered from the workshops that product testing seems to be a potential activity for this type of support, we currently keep an open direction to other design activities using scenarios as well. More design companies in addition to company $\mathrm{X}$ will be involved during the concept development. By observing various design practices, we aim to formulate a straightforward yet flexible support tool applicable for the differing design practices. Eventually, a software prototype will be developed to demonstrate the support tool and evaluate its practical feasibility.

\section{REFERENCES}

[1] Anggreeni, I. and van der Voort, M.C., Classifying Scenarios in a Product Design Process: a study towards semi-automated scenario generation. In: Proceedings of CIRP Design Conference 2008 (Enschede, The Netherlands, April 7-9, 2008).

[2] Beck, K. Extreme programming explained: embrace change. Addison-Wesley, 2000.

[3] Brouwer, M. and van der Voort, M.C., Design for Dynamic Use Situations. In Proceedings of Wonderground 2006 Design Research Society International Conference (Lisbon, Portugal, November 1-4, 2006).

[4] Carroll, J.M. Making Use: Scenario-Based Design of Human-Computer Interactions. MIT Press, London, 2000.

[5] Lim, Y. and Sato, K. Describing Multiple Aspects of Use Situation: Applications of Design Information Framework to Scenario Development. Design Studies, 27, 1 (2006). 57-76.

[6] Moggridge, B. Design by story-telling. Applied Ergonomics, 24, 1 (1993). 15-18.

[7] Rolland, C., Souveyet, C. and Achour, C.B. Guiding goal modelling using scenarios. IEEE Transactions on Software Engineering, Special Issue on Scenario Management, 24, 12 (1998). 1055-1071.

[8] Suri, J.F. and Marsh, M. Scenario building as an ergonomics method in consumer product design. Applied Ergonomics, 31, 2 (2000), 151-157. 